\title{
Pemphigus vulgaris - approach and management (Review)
}

\author{
IOANA ADRIANA POPESCU ${ }^{1}$, LAURA STATESCU $^{1,2}$, DAN VATA $^{1,2}$, ELENA PORUMB-ANDRESE $^{1,2}$, \\ ADRIANA IONELA PATRASCU ${ }^{1,2}$, IOANA-ALINA GRAJDEANU ${ }^{1}$ and LAURA GHEUCA SOLOVASTRU ${ }^{1,2}$ \\ ${ }^{1}$ Department of Dermatology, 'Grigore T. Popa' University of Medicine and Pharmacy, Faculty of Medicine, 700115 Iasi; \\ ${ }^{2}$ Dermatology Clinic, ‘St. Spiridon’ County Emergency Clinical Hospital, 700111 Iasi, Romania
}

Received June 11, 2019; Accepted August 6, 2019

DOI: $10.3892 /$ etm.2019.7964

\begin{abstract}
The place of pemphigus vulgaris (PV) among autoimmune bullous dermatoses is well known. In pemphigus, IgG autoantibodies are directed against desmogleins 1 and 3, which are part of the cadherin family of cell-cell adhesion molecules. These structures are responsible for maintaining the intercellular adherence in stratified squamous epithelia, such as the skin and oral mucosa. The incidence of autoimmune bullous dermatoses is steadily increasing, being associated with a high degree of morbidity. The pathophysiology of these dermatoses is very well understood, complemented by recent genetic studies. The gold standard for the diagnosis of pemphigus vulgaris is the detection of autoantibodies or complement component 3 by direct immunofluorescence microscopy of a perilesional biopsy. Early diagnosis and initiation of treatment are necessary in order to achieve a favorable prognosis. Although the first line of treatment is corticotherapy, there are no clear guidelines on dosing regimens, and long-term adverse effects are important. Corticosteroid-sparing adjuvant therapies have been employed in the treatment of PV, aiming to reduce the necessary cumulative dose of corticosteroids. In addition, therapies with anti-CD20 antibodies are used, but antigen-specific immune suppression-based treatments represent the future.
\end{abstract}

\section{Contents}

1. Introduction

2. Pathophysiology

3. Diagnosis

4. Treatment

5. Conclusions

Correspondence to: Dr Dan Vata, Department of Dermatology, 'Grigore T. Popa' University of Medicine and Pharmacy, Faculty of Medicine, Str. Universitatii nr. 16, 700115 Iasi, Romania

E-mail: danvata@yahoo.com

Key words: pemphigus, autoimmune bullous diseases, direct immunofluorescence microscopy, corticotherapy, azathioprine, rituximab

\section{Introduction}

Pemphigus vulgaris (PV) is a chronic, mucocutaneous, autoimmune bullous disease (1). The place of PV among autoimmune bullous dermatoses is well established. In pemphigus, IgG autoantibodies are directed against desmogleins 1 and 3, which are part of the cadherin family of cell-cell adhesion molecules. These structures are responsible for maintaining the intercellular adherence in stratified squamous epithelia, such as the skin and oral mucosa (2).

The word 'pemphigus' has its roots in the Greek 'pemphix', which means blister; Hippocrates first used it in 460-370 B.C. (3).

The estimated incidence of PV is between one and five cases per million every year. Its prevalence is higher in individuals of Ashkenazi Jewish, Mediterranean, Indian, Malaysian, Chinese and Japanese descent.

It is the most common subtype of pemphigus in Europe, the United States, and Japan; it usually affects women and most of the patients are 50-60 years of age. Though rare, some childhood cases have been reported $(4,5)$.

Usually, PV initially occurs with lesions on the oral mucosa, preceding skin lesions by several months. At this level, erosions with a small tendency to spontaneous, painful healing can be observed (6). The lesions start as blisters that rupture easily, causing erosions and ulcers, which can lead to serious life-threatening infections and metabolic abnormalities.

\section{Pathophysiology}

$\mathrm{PV}$ is one of the best-described autoimmune diseases; its pathophysiology seems to result from the deleterious actions of circulating auto-Abs, which are directed against desmosomal components, primarily desmoglein (Dsg3 and Dsg1), and lead to the loss of keratinocyte cell-cell adhesion within the epidermis, a phenomenon known as acantholysis (7). In addition, blister formation in PV was suggested to result from increased secretion of pro-inflammatory mediators or other mechanisms, such as activation of specific muscarinic receptors expressed by keratinocytes, abnormalities in intercellular signaling or activation of apoptosis (8-10).

Recent studies support the role of genetic factors in pemphigus. PV is a polygenic disease, and an increased prevalence of low titers of disease-associated autoantibodies in healthy first-degree relatives of patients with pemphigus 
has been reported $(10,11)$. The genetic contribution to the pathogenesis of PV is given by the association with other autoimmune diseases and from the ethnic clustering of PV. Pemphigus is linked to autoimmune thyroid disease, type I diabetes, rheumatoid arthritis, and systemic lupus erythematosus. In addition, the correlation between pemphigus and myasthenia gravis is well known (12). Several HLA alleles have been identified as risk factors, but the correlation between a certain HLA genetic profile and the patient's clinical profile is still unclear (13).

A meta-analysis demonstrated a strong connection between PV and HLA-DRB1_0402 (which is predominant in Ashkenazi Jews), HLA-DRB1_1401, HLA-DRB1_1404 and HLA-DQB1_0503 (which are both prevalent in non-Jewish patients of European and Asian descent) (14).

Environmental factors could be required to initiate and perpetuate the disease process. Thus, drugs, viral infections (herpes simplex virus), physical agents, contact allergens, vaccinations, dietary factors, and psychological stressors have been implicated in the disease (15).

The mechanism by which blisters are formed is related to the lack of adhesion of desmosomes and to specific cell signaling pathways $(16,17)$. Other studies have shown that pathogenic anti-Dsg monoclonal antibodies can bind directly to residues that mediate adhesion and that polyclonal antibodies contribute to acantholysis in a different way $(17,18)$.

\section{Diagnosis}

The diagnosis of PV is based on a combination of clinical presentation and detection of tissue-bound and/or circulating autoantibodies. The gold standard diagnostic of PV is direct immunofluorescence (DIF) microscopy, which can detect tissue-bound autoantibodies. In pemphigus, DIF microscopy reveals intercellular binding of $\operatorname{IgG}$ and/or C3 within the epidermis and/or epithelium (19).

Indirect immunofluorescence (IIF) is frequently used to semi-quantitatively measure circulating antibody levels, using monkey, rabbit or guinea pig esophagus as a substrate (19). The most frequently used substrates are monkey esophagus and human split skin.

Enzyme-linked immunosorbent assays (ELISA) are a more sensitive method for measuring antibodies to desmoglein 1 and desmoglein 3. ELISA reactivity correlates with disease activity; therefore, this test is useful both for diagnosis and for monitoring disease activity in patients with pemphigus (20). Immunoblot and immunoprecipitation techniques may be used to identify specific autoantibody profiles. These assays use recombinant proteins or extracts of dermis, epidermis or cultured keratinocytes $(18,21)$.

In addition, confocal laser scanning microscopy is a non-invasive tool for the diagnosis of pemphigus and differentiation of its subtype; it points to the need for further investigation of the patient. It has the advantage of monitoring disease progression and the treatment efficacy $(22,23)$.

\section{Treatment}

Without treatment, PV has a mortality rate ranging from 60 to $90 \%$ (24). Moreover, multiple life-threatening complications may occur, such as sepsis, fluid and electrolyte imbalances, impaired thermoregulation, as well as cardiac and renal failure. Systemic corticosteroids and adjuvant therapies have reduced the mortality rate of PV patients to approximately $10 \%$ (24).

The role of treatment in patients with PV is to suppress the immune system and to prevent the production of pathogens. The immunological response is due to the significant decrease of pathogens in the skin and serum of patients, which clinically corresponds to the cessation of new vesicle formation and the maintenance of remission (25). Given the rarity of this disease, there are few evidence-based controlled studies in literature regarding the safety and efficacy of the therapeutic interventions for PV.

The British guidelines recommend the consideration of two stages in the management of PV: induction of remission and maintenance of remission. Thus, in the first stage, the treatment is represented by corticosteroids, less than adjuvant therapy, until $80 \%$ of the lesions heal and for 2 weeks no new lesions will appear, both cutaneous and mucosal. The remission maintenance phase is about gradual dose reduction in order to achieve effective pathology control. The goal of treatment is to keep the remission as long as possible. Following an international consensus, the $10 \mathrm{mg}$ daily dose of prednisolone was established (26) (Table I).

The European and Japanese guidelines include corticosteroids and immunosuppressive reagents, such as azathioprine and cyclophosphamide, plasmapheresis, intravenous immunoglobulin (IVIG), and rituximab $(27,28)$.

The first-line treatment is represented by corticosteroids, owing to their rapid effect (within days). In refractory cases, Japanese guidelines recommend short-term high-dose of intravenous methylprednisolone, an approach that is supported by retrospective studies (27).

When corticosteroids are administered over long periods of time, side effects such as diabetes, hypertension or osteoporosis may occur. It is preferable to combine systemic corticosteroids with an immunosuppressive agent, in order to avoid side effects $(28,29)$. The speed with which corticosteroid takes effect is attributed to increased transcription of desmogleins and other cell adhesion molecules, which counteracts the autoantibody-induced interference with desmoglein adhesive function (29). These multiple adverse events may even contribute to poor prognostic outcomes and death, and for that purpose, many studies have attempted to identify the minimum dose required to induce and maintain remission (29).

Azathioprine and mycophenolate mofetil represent the first-line adjunctive immunosuppressive therapies in pemphigus, considered safe and efficient (30). Azathioprine is likely the most commonly used steroid-sparing agent worldwide. Azathioprine demonstrated a steroid-sparing effect when compared to prednisolone alone (31), while mycophenolate mofetil has shown faster and more-durable treatment responses than placebo when added to prednisone regimens (32). The recommended dosage of Azathioprine in PV is $100-200 \mathrm{mg} /$ day (1-3 mg/kg/day), administered orally. Its main side effects are leukopenia, thrombocytopenia, anemia, pancytopenia and hepatotoxicity. Mycophenolate mofetil presents lower hepatotoxicity and is more effective in controlling PV than azathioprine (33). 
Table I. Therapeutic options for the treatment of pemphigus vulgaris.

Therapy Method

First-line therapy

Second-line therapy

Third-line therapy
Corticosteroids (Prednisolone)

Start with $1 \mathrm{mg} / \mathrm{kg}$ per day in severe cases

$0.5-1 \mathrm{mg} / \mathrm{kg}$ per day in milder cases

Doses may be increased by 50-100\% every 5-7 days if blistering continues

After installation of the remission period, the doses are gradually decreased

(5-10 $\mathrm{mg}$ prednisolone/2 weeks down to $20 \mathrm{mg}$ daily, then by $2-5 \mathrm{mg}$ every

2-4 weeks down to $10 \mathrm{mg}$ daily)

Add an adjuvant immunosuppressant:

Azathioprine 2-3 mg kg - 1 per day

Mycophenolate mofetil 2-3 g per day

Rituximab (rheumatoid arthritis protocol, $291 \mathrm{~g}$ infusions, 2 weeks apart)

If first-line treatment does not work, switch to alternate

corticosteroid-sparing agent (azathioprine, mycophenolate mofetil or rituximab)

Cyclophosphamide

Immunoadsorption

Intravenous immunoglobulin

Methotrexate

Plasmapheresis or plasma exchange

Adapted from British Association of Dermatologists' guidelines for the management of pemphigus vulgaris 2017 (26).

Cyclophosphamide is an alkylating agent, used like adjuvant therapy in PV. It can be administered orally (1-3 mg/kg/day) or intravenously, with or without pulse corticotherapy. Because it has adverse events such as infertility, lymphopenia, predisposition to neoplasia, it is recommended for use only in refractory cases of mycophenolate mofetil or azathioprine (33).

Dapsone is a drug with anti-inflammatory and anti-TNF activity that can be tried as adjuvant medication in PV. It can be administered orally at $50-200 \mathrm{mg} / \mathrm{day}$, as an adjuvant to corticotherapy in the maintenance phase of the disease. Its side effects are usually dose-dependent and reversible $(26,33)$.

Methotrexate (MTX) is a chemotherapeutic and immunosuppressive agent; the first case series on MTX in PV was published in 1969. In 2012, a retrospective review of methotrexate use in PV showed the efficacy of methotrexate as adjunctive therapy in moderate-severe cases of pemphigus. In the study, 30 patients were included, who were evaluated by disease severity score and prednisone dosage. The dosage of methotrexate was $15 \mathrm{mg}$ per week. Only four patients presented mild side effects; methotrexate treatment was proven safe and effective in the other patients (34).

Cyclosporine is a calcineurin inhibitor with potent immunosuppressive activity against $\mathrm{B}$ and $\mathrm{T}$ lymphocytes. It is rarely used as an adjuvant in the treatment of $\mathrm{PV}$ at dosages of 3 to $5 \mathrm{mg} / \mathrm{kg} /$ day. Olszewska et al reported a retrospective series of 101 patients with PV, in which cyclosporine-treated patients showed no improvement in the overall condition (35).

Recent studies demonstrated advantages of rituximab, a monoclonal anti-CD20 antibody that targets CD20 ${ }^{+}$B cells, in the treatment of pemphigus $(25,36)$. A randomized trial of rituximab was recently performed in France, as a first-line treatment for moderate to severe cases of pemphigus. Two years after treatment, 41 (89\%) out of the 46 patients assigned to rituximab plus short-term prednisone were in complete remission and off therapy versus 15 (34\%) out of the 44 patients assigned to the prednisone-alone treatment. In the rituximabplus-prednisone group, less adverse effects were reported than in a prednisone-alone group. Thus, the administration of Rituximab in patients with pemphigus has been shown to be far more reliable than corticotherapy. The treatment with rituximab could be considered as the first-line in pemphigustreated patients (36).

Intravenous immunoglobulins (IVIG) neutralize and slow down the production of circulating pemphigus antibodies. They are considered an adjuvant therapy for pemphigus. Patients treated with intravenous immunoglobulin are not at high risk of infection or reactivation of chronic infections, as compared to those under conventional immunosuppressants. IVIG are effective at a dosage of $0.4 \mathrm{~g} / \mathrm{kg} /$ day for 5 days, used as an adjunct to corticosteroid therapy once per month (33). In a randomized, double-blind, placebo-controlled trial, the efficacy of intravenous immunoglobulin to pemphigus patients was evaluated. It was proven that the therapeutic response of patients receiving immunoglobulin was much better than those in the placebo group (37). In addition, this therapy is considerated the safest adjuvant treatment for pemphigus in pregnant women (38).

Plasmapheresis involves plasma exchange with albumin or fresh frozen plasma to remove serum IgG antibodies. This procedure has been used to treat a variety of antibodymediated autoimmune disorders. This therapeutic alternative is generally used in patients who experience side effects 
in cortisone therapy, pregnant women or those who do not respond to conventional therapies $(26,39)$.

Guillaume et al studied the role of plasmapheresis in a multicenter randomized trial of 40 pemphigus patients. The outcome highlighted that plasmapheresis in association with low steroid doses versus the steroid-treated group did not show any additional benefit in the treatment of pemphigus. Moreover, 4 out of 22 patients in the intervention group died of thromboembolism or infection (39).

Immunoadsorption is more efficient and safer than plasmapheresis and can be used for the treatment of severe, recalcitrant $\mathrm{PV}$. This procedure leads to the rapid removal of circulating autoantibodies against desmoglein 1 and desmoglein 3, only $\operatorname{IgGs}$ and immune complexes are attracted to the absorber and removed from the circulation. Some studies have highlighted that immunoadsorption is more effective when used in combination with immunosuppressive agents, including corticosteroids, azathioprine, and rituximab $(40,41)$.

The perfect therapy for pemphigus should eliminate pathogenic autoimmune cells while sparing protective immunity, but feasible strategies for such an approach have been elusive. Recent studies demonstrated that Veltuzumab, a humanized anti-CD20 monoclonal antibody, provided the best results when used for patients with refractory pemphigus (42). Other researchers revealed the therapy through the use of chimeric antigen receptor $\mathrm{T}$ cells. Therefore, it would directly eliminate anti-Dsg3 memory B cells and indirectly eliminate Dsg3-specific short-lived plasma cells that produce diseasecausing antibodies (43).

These new approaches represent an ideal therapeutic strategy because the target antigens and pathophysiological mechanisms of pemphigus are well known.

Thus, the first-line treatment of moderate and severe cases of PV, based on literature and the personal experience of the authors is represented by systemic corticosteroid therapy (prednisone or prednisolone). In refractory cases or with contraindications to corticosteroid therapy, treatment with immunosuppressive agents may be prescribed (azathioprine, mycophenolate mofetil, methotrexate, cyclophosphamide) to minimize side effects. The treatment for the severe and nonresponsive cases may be represented by corticosteroids in the form of intravenous pulse therapy, plasmapheresis and IVIG $(33,43)$.

\section{Conclusions}

Although they are considered rare diseases, the incidence of autoimmune bullous dermatoses is steadily increasing, being associated with a high degree of morbidity and occasional mortality. The cutaneous damage, such as blister formation, pain, itch, and associated functional limitations have a psychoemotional impact on patients and can severely affect the patient's quality of life. Pemphigus is a life-threatening disorder for which early recognition and treatment are necessary in order to achieve a favorable prognosis.

The gold standard for the diagnosis of PV is the detection of autoantibodies or complement component 3 by direct immunofluorescence microscopy of a perilesional biopsy. Early diagnosis and initiation of treatment are compulsory for a promising prognosis, and a multidisciplinary approach is recommended to optimize patient care. Systemic corticosteroids represent the first-line of treatment, though their optimal dosing regimen remains unknown. There are many therapeutic alternatives, but treatment needs to be tailored to each patient. The introduction of biologic therapy into the therapeutic algorithm has the potential to improve overall outcomes and reduce drug-side effects. However, future could be represented by antigen-specific immune suppression-based treatments as researchers continue to develop novel targeted therapies for the treatment of PV.

\section{Acknowledgements}

Professional editing, linguistic and technical assistance performed by Individual Service Provider Irina Radu, certified translator in Medicine and Pharmacy.

\section{Funding}

No funding was received.

\section{Availability of data and materials}

Not applicable.

\section{Authors' contributions}

IAP conceived this review article. LGS and DV were responsible for the collection and assembly of the articles/published data, for inclusion and interpretation in this review. LS, EPA, IAG and AIP were responsible for the data acquisition. All authors contributed to the acquisition of the data and critical revision of manuscript for important intellectual content. All authors read and approved the final version of the manuscript.

\section{Ethics approval and consent to participate}

Not applicable.

\section{Patient consent for publication}

Not applicable.

\section{Competing interests}

The authors declare that they have no competing interests.

\section{References}

1. Chaidemenos G, Apalla Z, Koussidou T, Papagarifallou I and Ioannides D: High dose oral prednisone vs. prednisone plus azathioprine for the treatment of oral pemphigus: A retrospective, bi-centre, comparative study. J Eur Acad Dermatol Venereol 25: 206-210, 2011.

2. Burgan SZ, Sawair FA and Napier SS: Case report: Oral pemphigus vulgaris with multiple oral polyps in a young patient. Int Dent J 53: 37-40, 2003.

3. Lever WF and Talbott JH: Pemphigus: A historical study. Arch Derm Syphilol 46: 800-823, 1942.

4. Alpsoy E, Akman-Karakas A and Uzun S: Geographic variations in epidemiology of two autoimmune bullous diseases: Pemphigus and bullous pemphigoid. Arch Dermatol Res 307: 291-298, 2015. 
5. Shah AA, Seiffert-Sinha K, Sirois D, Werth VP, Rengarajan B, Zrnchik W, Attwood K and Sinha AA: Development of a disease registry for autoimmune bullous diseases: Initial analysis of the pemphigus vulgaris subset. Acta Derm Venereol 95: 86-90, 2015

6. Ramos-e-Silva M, Ferreira A and Jacques C: Oral involvement in autoimmune bullous diseases. Clin Dermatol 29: 443-454, 2011.

7. Stanley JR and Amagai M: Pemphigus, bullous impetigo, and the staphylococcal scalded-skin syndrome. N Engl J Med 355: 1800-1810, 2006.

8. Grando SA: Pemphigus autoimmunity: Hypotheses and realities. Autoimmunity 45: 7-35, 2012.

9. Grando SA, Bystryn JC, Chernyavsky AI, Frusić-Zlotkin M, Gniadecki R, Lotti R, Milner Y, Pittelkow MR and Pincelli C: Apoptolysis: A novel mechanism of skin blistering in pemphigus vulgaris linking the apoptotic pathways to basal cell shrinkage and suprabasal acantholysis. Exp Dermatol 18: 764-770, 2009.

10. Ahmed AR, Carrozzo M, Caux F, Cirillo N, Dmochowski M, Alonso AE, Gniadecki R, Hertl M, López-Zabalza MJ, Lotti R, et al: Monopathogenic vs multipathogenic explanations of pemphigus pathophysiology. Exp Dermatol 25: 839-846, 2016.

11. Torzecka JD, Woźniak K, Kowalewski C, Waszczykowska E, Sysa-Jedrzejowska A, Pas HH and Narbutt J: Circulating pemphigus autoantibodies in healthy relatives of pemphigus patients: Coincidental phenomenon with a risk of disease development? Arch Dermatol Res 299: 239-243, 2007.

12. Ruocco E, Wolf R, Ruocco V, Brunetti G, Romano F and Lo Schiavo A: Pemphigus: associations and management guidelines: facts and controversies. Clin Dermatol 31: 382-390, 2013.

13. Vodo D, Sarig O and Sprecher E: The genetics of pemphigus vulgaris. Front Med (Lausanne) 5: 226, 2018.

14. Ruocco V, Ruocco E, Lo Schiavo A, Brunetti G, Guerrera LP and Wolf R: Pemphigus: etiology, pathogenesis, and inducing or triggering factors: facts and controversies. Clin Dermatol 31 : 374-381, 2013

15. Mao X, Sano Y, Park JM and Payne AS: p38 MAPK activation is downstream of the loss of intercellular adhesion in pemphigus vulgaris. J Biol Chem 286: 1283-1291, 2011.

16. Saito M, Stahley SN, Caughman CY, Mao X, Tucker DK, Payne AS, Amagai M and Kowalczyk AP: Signaling dependent and independent mechanisms in pemphigus vulgaris blister formation. PLoS One 7: e50696, 2012

17. Yamagami J: Recent advances in the understanding and treatment of pemphigus and pemphigoid. F1000Res pii: F1000, 2018.

18. Witte M, Zillikens D and Schmidt E: Diagnosis of autoimmune blistering diseases. Front Med (Lausanne) 5: 296, 2018.

19. Shoimer I, Wong RX and Mydlarski PR: Pemphigus Vulgaris In: Autoimmune Bullous Diseases. Sami N (ed). Springer International Publishing, pp1-18, 2016

20. Hammers CM, Chen J, Lin C, Kacir S, Siegel DL, Payne AS and Stanley JR: Persistence of anti-desmoglein $3 \mathrm{IgG}(+)$ B-cell clones in pemphigus patients over years. J Invest Dermatol 135 742-749, 2015.

21. Xuan RR, Yang A and Murrell DF: New biochip immunofluorescence test for the serological diagnosis of pemphigus vulgaris and foliaceus: A review of the literature. Int J Womens Dermatol 4: 102-108, 2018

22. Ilie MA, Caruntu C, Lupu M, Lixandru D, Tampa M, Georgescu SR, Bastian A, Constantin C, Neagu M, Zurac SA, et al: Current and future applications of confocal laser scanning microscopy imaging in skin oncology. Oncol Lett 17: 4102-4111, 2019.

23. Ilie MA, Caruntu C, Lixandru D, Tampa M, Georgescu SR, Constantin MM, Constantin C, Neagu M, Zurac SA and Boda D: In vivo confocal laser scanning microscopy imaging of skin inflammation: Clinical applications and research directions. Exp Ther Med 17: 1004-1011, 2019.

24. Bystryn JC and Rudolph JL: Pemphigus. Lancet 366: 61-73, 2005.

25. Kridin K: Emerging treatment options for the management of pemphigus vulgaris. Ther Clin Risk Manag 14: 757-778, 2018.

26. Harman KE, Brown D, Exton LS, Groves RW, Hampton PJ, Mohd Mustapa MF, Setterfield JF and Yesudian PD: British Association of Dermatologists' guidelines for the management of pemphigus vulgaris. Br J Dermatol 177: 1170-1201, 2017.

27. Amagai M, Tanikawa A, Shimizu T, Hashimoto T, Ikeda S, Kurosawa M, Niizeki H, Aoyama Y, Iwatsuki K and Kitajima Y; Committee for Guidelines for the Management of Pemphigus Disease: Japanese guidelines for the management of pemphigus. J Dermatol 41: 471-486, 2014.
28. Hertl M, Jedlickova H, Karpati S, Marinovic B, Uzun S, Yayli S, Mimouni D, Borradori L, Feliciani C, Ioannides D, et al: Pemphigus. S2 Guideline for diagnosis and treatment - guided by the European Dermatology Forum (EDF) in cooperation with the European Academy of Dermatology and Venereology (EADV). J Eur Acad Dermatol Venereol 29: 405-414, 2015.

29. Caplan A, Fett N, Rosenbach M, Werth VP and Micheletti RG: Prevention and management of glucocorticoid-induced side effects: A comprehensive review: A review of glucocorticoid pharmacology and bone health. J Am Acad Dermatol 76: 1-9, 2017.

30. Almugairen N,Hospital V, Bedane C, Duvert-Lehembre S,Picard D, Tronquoy AF, Houivet E, D'incan M and Joly P: Assessment of the rate of long-term complete remission off therapy in patients with pemphigus treated with different regimens including medium- and high-dose corticosteroids. J Am Acad Dermatol 69: 583-588, 2013.

31. Chams-Davatchi C, Mortazavizadeh A, Daneshpazhooh M, Davatchi F, Balighi K, Esmaili N, Akhyani M, Hallaji Z, Seirafi $\mathrm{H}$ and Mortazavi H: Randomized double blind trial of prednisolone and azathioprine, vs. prednisolone and placebo, in the treatment of pemphigus vulgaris. J Eur Acad Dermatol Venereol 27: 1285-1292, 2013.

32. Beissert S, Mimouni D, Kanwar AJ, Solomons N, Kalia V and Anhalt GJ: Treating pemphigus vulgaris with prednisone and mycophenolate mofetil: A multicenter, randomized, placebocontrolled trial. J Invest Dermatol 130: 2041-2048, 2010.

33. Porro AM, Hans Filho G and Santi GH: Consensus on the treatment of autoimmune bullous dermatoses: Pemphigus vulgaris and pemphigus foliaceus - Brazilian Society of Dermatology. An Bras Dermatol 94 (Suppl 1): 20-32, 2019.

34. Baum S, Greenberger S, Samuelov L, Solomon M, Lyakhovitsky A, Trau H and Barzilai A: Methotrexate is an effective and safe adjuvant therapy for pemphigus vulgaris. Eur J Dermatol 22: 83-87, 2012

35. Olszewska M, Kolacinska-Strasz Z, Sulej J, Labecka H, Cwikla J, Natorska U and Blaszczyk M: Efficacy and safety of cyclophosphamide, azathioprine, and cyclosporine (ciclosporin) as adjuvant drugs in pemphigus vulgaris. Am J Clin Dermatol 8: 85-92, 2007.

36. Joly P, Maho-Vaillant M, Prost-Squarcioni C, Hebert V, Houivet E, Calbo S, Caillot F, Golinski ML, Labeille B, Picard-Dahan $\mathrm{C}$, et al; French study group on autoimmune bullous skin diseases: First-line rituximab combined with shortterm prednisone versus prednisone alone for the treatment of pemphigus (Ritux 3): A prospective, multicentre, parallel-group, open-label randomised trial. Lancet 389: 2031-2040, 2017.

37. Amagai M, Ikeda S, Shimizu H, Iizuka H, Hanada K, Aiba S, Kaneko F, Izaki S, Tamaki K, Ikezawa Z, et al; Pemphigus Study Group: A randomized double-blind trial of intravenous immunoglobulin for pemphigus. J Am Acad Dermatol 60: 595-603, 2009.

38. Tavakolpour S, Mirsafaei HS and Delshad S: Management of pemphigus disease in pregnancy. Am J Reprod Immunol 77: e12601, 2017

39. Guillaume JC, Roujeau JC, Morel P, Doutre MS, Guillot B, Lambert D, Lauret P, Lorette G, Prigent F, Triller R, et al: Controlled study of plasma exchange in pemphigus. Arch Dermatol 124: 1659-1663, 1988.

40. Kolesnik M, Becker E, Reinhold D, Ambach A, Heim MU, Gollnick $\mathrm{H}$ and Bonnekoh B: Treatment of severe autoimmune blistering skin diseases with combination of protein A immunoadsorption and rituximab: A protocol without initial high dose or pulse steroid medication. J Eur Acad Dermatol Venereol 28: 771-780, 2014.

41. Kasperkiewicz M, Shimanovich I, Meier M, Schumacher N, Westermann L, Kramer J, Zillikens D and Schmidt E: Treatment of severe pemphigus with a combination of immunoadsorption, rituximab, pulsed dexamethasone and azathioprine/mycophenolate mofetil: A pilot study of 23 patients. Br J Dermatol 166: 154-160, 2012.

42. Ellebrecht CT, Choi EJ, Allman DM, Tsai DE, Wegener WA, Goldenberg DM and Payne AS: Subcutaneous veltuzumab, a humanized anti-CD20 antibody, in the treatment of refractory pemphigus vulgaris. JAMA Dermatol 150: 1331-1335, 2014.

43. Ellebrecht CT, Bhoj VG, Nace A, Choi EJ, Mao X, Cho MJ, Di Zenzo G, Lanzavecchia A, Seykora JT, Cotsarelis G, et al: Reengineering chimeric antigen receptor $\mathrm{T}$ cells for targeted therapy of autoimmune disease. Science 353: 179-184, 2016.

(i) $($ This work is licensed under a Creative Commons Attribution-NonCommercial-NoDerivatives 4.0 International (CC BY-NC-ND 4.0) License. 